\title{
Pointing Sensors and Sun Tracking Techniques
}

\author{
D. Fontani, P. Sansoni, F. Francini, D. Jafrancesco, L. Mercatelli, and E. Sani
}

CNR-INO Istituto Nazionale di Ottica, Largo E. Fermi 6, 50125 Firenze, Italy

Correspondence should be addressed to P. Sansoni, paola.sansoni@ino.it

Received 5 November 2010; Revised 20 February 2011; Accepted 3 March 2011

Academic Editor: Roel van de Krol

Copyright () 2011 D. Fontani et al. This is an open access article distributed under the Creative Commons Attribution License, which permits unrestricted use, distribution, and reproduction in any medium, provided the original work is properly cited.

\begin{abstract}
Every optical system for sunlight concentration requires following the sun in its movement. The sun tracking method is essentially chosen on the base of collection geometry and optical system configuration. A simple, useful, and original technique to realise sun tracking is proposed. It is based on a double guiding system using two complementary procedures. A passive tracking device performs a preliminary collector orientation. Then an active tracking system realises its fine positioning and adjustments exploiting an optical pointing sensor. The core of this active tracking device is the sun finder. Pointing sensors for fibre-coupled, CPV (Concentrating Photo voltaic), and linear collectors are presented, illustrating in detail the working principle and practical use. All sensors were optically characterised in laboratory, under controlled and reproducible conditions. Some field tests completed the experimentation evaluating the sensors performance in outdoor working conditions.
\end{abstract}

\section{Introduction}

Tracking the movement of the sun is a strategy to enhance the performance of solar photovoltaic plants. Using plane Photo voltaic (PV) panels, the required pointing precision is of the order of few angular degrees; hence it is sufficient to drive the motors dedicated to orient the PV panels, using a software taking into account sun's ephemerides during the arc of the year [1-6]. More simplified guiding systems rotate the solar panel only in one direction, during the whole day, while the inclination over the horizon is kept constant and equal to the mean value of solar annual elevation.

In Concentrating PhotoVoltaic (CPV) plants that, on the contrary, can work only with direct sunlight, it is necessary to employ an optical pointing system. In these devices, the central optical component typically is a Fresnel lens or a parabolic concentrator and some secondary optical elements could be added to improve pointing precision and plant collection efficiency.

Maintaining aligned the optical system with the sun's position requires an angular precision higher than the tenth of degree. This accuracy is not realisable using a passive driving device due to problems connected to the plant orientation, difficulties in keeping a high mechanical stability, wind action, and the precision required by the type of concentrator in use. For these reasons, it is not sufficient to use only the alignment technique based on the ephemerides; so the solar plants usually employ an additional device devoted to supply to the moving system the information necessary to correct the concentrators' orientation in order to keep them aligned in the sun's direction.

These devices, used to furnish the error signal to the control electronics, are commonly called "sun finders". In general, the optoelectronic device utilises couples of photodetectors that, in conditions of alignment in the sun's direction, supply the same response because they detect the same amount of light. The simplest device is composed of an opaque sector, which separates two photodiodes [7]. In alternative, other optical configurations can be used to project shadows on the detectors: for instance, two crossed opaque sectors or a rod centred on a screen (like in a sundial) [8].

However, an enhanced pointing accuracy can be obtained employing an optical system with a focal length $F$, which projects the sun's image on the photodetectors. In particular, the angular precision of the sun tracking improves as the focal length $F$ increases. Considering that having to observe the sun there are no problems of luminous intensity, the optical system can be replaced with a tiny pinhole ("pinhole camera") [9-14]. Sun finders equipped with an optical system are also utilised as astronomical pointing devices [15]. 


\section{Sun's Altitude and Collector Placement for Sun Tracking}

The movement of the sun with respect to a determined site on the Earth surface seems very complicated. The sun's altitude over the horizon depends on the site Latitude and it varies during the year and during the day. Figure 1 illustrates the variations of sun's altitude for the Latitude of Firenze (Italy), located at Latitude of $43.75^{\circ}$ North. The sun's altitude $\alpha$ is reported versus solar time (in hours), from $6 \mathrm{AM}$ to $6 \mathrm{PM}$, considering four representative months. Each month curve is obtained averaging the values corresponding to all the days composing the month. The maximum value of solar rays inclination, with respect to the vertical direction $(\alpha=$ $90^{\circ}$ ), is achieved in December; the minimum value is reached in June. For Firenze, the incidence angle of sun's rays varies from $67^{\circ}$ (for $\alpha=23^{\circ}$ in December) to $21^{\circ}$ (for $\alpha=69^{\circ}$ in June).

The movement of the sun can be subdivided in two components. These two movements correspond to the sun tracking for a linear collector in the North-South and EastWest placements. Then the bidirectional tracking system combines the two movement components to follow the sun's displacement during the day and for each day of the year.

If the linear collector axis is parallel to the North-South direction, the tracking system must follow the sun in its daily excursion and the sun's altitude over the horizon depends on Latitude and day of the year. With this layout, the solar rays impinge on the collector with an inclination dictated by Latitude and hour of the day.

If the linear collector axis is parallel to the East-West direction, the sun should not be tracked in its daily excursion. However, the tracking system must follow the displacements in sun's altitude occurring every day of the year. In this layout only at noontime sun's rays are perpendicular to the entrance aperture of the solar trough.

Linear tracking is obviously employed for sunlight collecting systems working in one direction, like solar troughs or linear lenses. Linear mirrors of troughs are typically combined to linear metal pipes for thermal or cooling applications, but the absorber can also be constituted by an array of PV cells. Linear lenses concentrators are mostly made of Fresnel lenses and they can either be used in thermal or low concentration CPV applications.

The bidirectional tracking is applied to solar collectors working on two axes, like point focus systems or dish systems. These collection systems are mostly coupled to PV cells of various size, shape, and features, which sometime require the introduction of a secondary optics. However, these solar concentrators could also be coupled to optical fibres, exploiting the same tracking techniques. In the bidirectional tracking, the optical collectors are oriented in the sun's direction using an equatorial structure with two movements on the temporal axis and on the declination axis. The system should be able to compensate possible errors in the applications of the device, which must be positioned with the temporal axis parallel to the Earth axis.

The accurate placement of the sunlight collection device is the essential requirement for the efficacy of any tracking

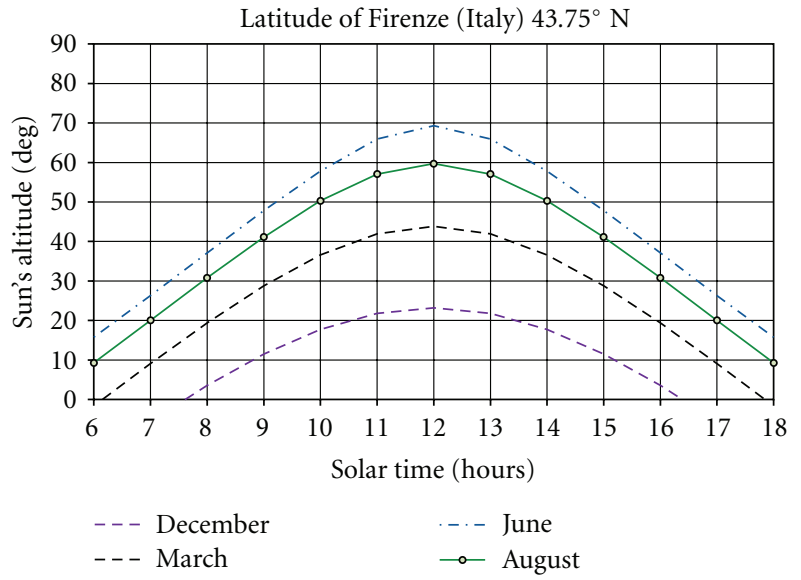

Figure 1: Altitude of sun over the horizon for Firenze (Italy).

technique. The movements, the necessary precision level and the orientation actuation are facilitated by a precise solar plant positioning. However, placement errors can be compensated modifying the angle of tracking system rotation. This angular correction corresponds to the daily sun tracking. The values of correction angle are experimentally determined maximising the light received by the absorber. For example, for thermal linear collectors a misalignment error in North-South placement can be recovered up to $15^{\circ}$, typically with negligible energy losses [16].

This practical compensation could also mitigate the effects of mechanical misalignments or tracking errors or external agents (atmospheric or environmental). However, its efficacy basically depends on collection system and on orientation errors amount: only after examining the specific case, it can be assessed how much the correction method is able to compensate the orientation errors. Finally the compensation effectiveness also depends on sun's altitude $\alpha$ [16].

\section{Tracking Techniques Using the Sun Finder}

The proposed tracking methodology consists in using two complementary tracking techniques, which are integrated to keep the optical collector oriented in the sun's direction. Hence the device for collector movement is provided with a double guiding system. The first one is of passive type and it drives the motors in order to correctly orient the collector every day of the year, while the second one is of dynamic type and it exploits an optical sun finder. The two tracking techniques are complementary since the first one provides the preliminary orientation, then the second one realises the fine positioning and adjustments. The passive preliminary orientation is necessary to track the sun position when the solar light does not reach the sun finder (in case of could passage or sun absence). In this way when the sun illuminates again the sun finder, the active system can take the control of thes tracking. 
The advantage of the double-driving technique is to confer flexibility to the tracking system, which automatically follows every weather variation in all environmental conditions. To achieve and maintain the sun tracking, the double guiding system must fulfil several requirements. The time axis of the device should to be oriented in the North-South direction; nevertheless, the device should correctly work even in case of an approximate placement. During the starting phase, the optical system must reach the correct orientation in the sun's direction, even in cloudy sky conditions. The speed of motors ought to be synchronised with the sun's velocity. Therefore, the value of set speed should consider geographic position, collector inclination, and day of the year. When the cloudy sky comes back to a clear sky condition, the sun's image will be aligned with the photodetector only if the motor speed is appropriate and the initial positioning operation is correctly carried out. On the contrary (the more frequent case), the control is taken by the sun finder.

The sun finder is the optical device constituting the active guiding system of the tracking methodology. To improve the pointing precision, we suggest the use of a device including two sections, with different Field Of View apertures (FOV), corresponding to different accuracies. The low-precision section allows the reorientation of the collector within a suitably large FOV, while the high-precision section has a smaller FOV. The two sections of the sun finder are used in sequence, improving the accuracy. The FOVs are determined by the solar application and usually the larger FOV exceeds $30^{\circ}$, while the smaller FOV is lower than $10^{\circ}$.

Two examples of realised sun finders are shown in Figures 2 and $3(a)$; then Figure $3(b)$ reports the scheme of the sun finder in Figure 3(a). The pointer in Figures 3(a) and 3(b) includes two sections mounting Pinhole Sensors (described in Section 4). The low-precision section has an FOV of $40^{\circ}$ and it is the right one in both Figures 3(a) and 3(b). The more accurate one has an FOV of $7^{\circ}$, and it is the left section in Figures 3(a) and 3(b). In synthesis, this optical pointing device works as a double pinhole camera without lenses.

Figure 3(b) schematically illustrates the two sections of the sun finder: each section is composed of a well with a pinhole on the top and a four-quadrant detector on the bottom. The distance $L$ from the pinhole to the four-quadrant detector determines the Field Of View of the pointer section. Sun image dimension and light intensity on the detector depend on the diameter $d$ of the pinhole. The value of the distance $L$ is $42 \mathrm{~mm}$ for the high-precision section, which corresponds to $\mathrm{FOV}=7^{\circ}$. The diameter $d$ of the pinhole is $0.8 \mathrm{~mm}$ and it has been experimentally determined. For the low-precision section, $L=7 \mathrm{~mm}$ and the resulting FOV is $40^{\circ}$. In both cases, the squared four-quadrant detector measures $5 \mathrm{~mm}$ of side. At the base of the two wells, forming the cameras, there are two boards mounting the four-quadrant detectors and their preamplifiers with gain adjustments. Figure 3(a) depicts the sun finder device without the two pinholes covering the four-quadrant detectors. The third aperture, visible in the picture, allows a visual alignment control during the assembly phase, in particular to correctly and precisely place the detectors.

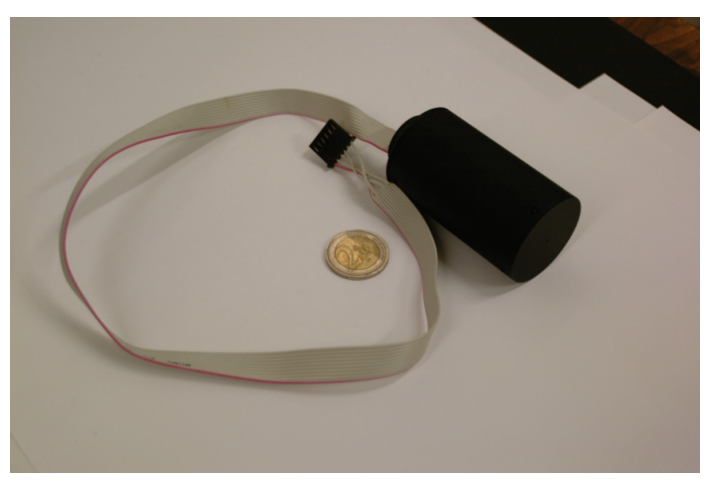

Figure 2: Example of sun finder with 1 section.

\section{Sun Finders for High Concentration Tracking}

In the last decade, Concentrating Photovoltaics (CPV systems) have improved their diffusion and the related electronics, and optical components have experienced great technological and scientific developments. Photovoltaic cells of the last generation require concentrations that can exceed 700 suns. Consequently, the associated optical systems must have a quite high value of posterior numerical aperture and a very tiny Field Of View. These requirements impose a very high angular precision to the systems for sun tracking. For tracking sensors that guide high concentration systems, the typical required resolution is at least $0.1^{\circ}$.

Sun tracking has been studied and experimented in our laboratory since 1997, developing mechanics, electronics, and software to realise prototypes [9-14]. Several possibilities for the sun pointing sensor have been examined. The paper illustrates two examples of optical pointing sensors, comparing optical characteristics and performance. The Pinhole Sensor is essentially a pinhole camera with no lens, while the Slit Sensor is basically a photodetector coupled to a slit. The Pinhole Sensor works on two axes, so it is suitable for fibre-coupled or CPV collectors. The Slit Sensor works on one axis, hence it is appropriate for solar troughs or linear lenses. The working principle is the same for both sensors.

All sensors were tested in laboratory under controlled and reproducible conditions. This laboratory experimentation includes a deep analysis of angular resolution and Field Of View of the sensors. The purpose of these tests is to provide an optical characterisation of the devices. Finally the sensors were tested under operative conditions in an outdoor installation.

The Pinhole Sensor works as a pinhole camera without lenses. Figure 4 shows the main elements composing the Pinhole Sensor: pinhole and four-quadrant detector (4Qdetector).

The distance $L$, between pinhole and four-quadrant detector, determines the sensor Field Of View. $L$ corresponds to the focal length of the collecting optics, hence the sun's image moves on the photodetector with the same speed of the image on the PV cell. Sun's image dimension and light intensity on the detector depend on the pinhole diameter $d$. The angular resolution of the sensor depends on detector 


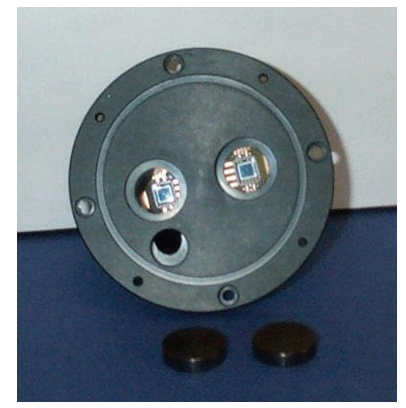

(a)

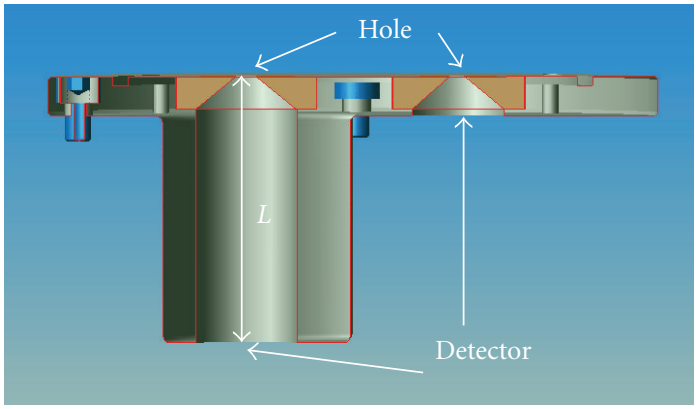

(b)

Figure 3: (a) A sun finder with 2 sections. (b) Scheme of the pointer in (a).

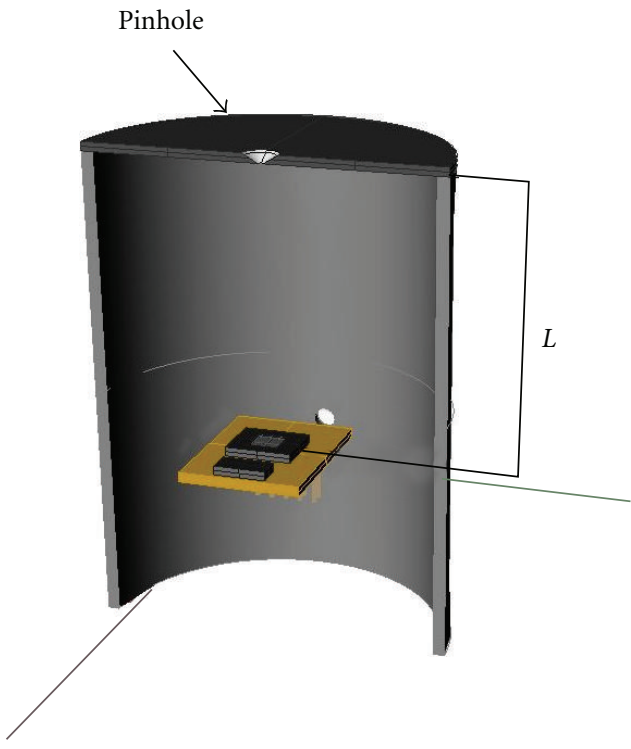

FIgURe 4: Pinhole sensor.

dimensions, pinhole size, and pinhole-detector distance. The lateral dimension $h$ of the square photodetector is few millimetres.

The FOV aperture can be obtained as

$$
\mathrm{FOV}=2 \operatorname{arctg} \frac{h}{2 * L} .
$$

For $L=50 \mathrm{~mm}$ and $h=5 \mathrm{~mm}$, the total FOV angle is $5.7^{\circ}$.

The Slit Sensor is analogous to the Pinhole Sensor, with a slit replacing the pinhole. Consequently, the four-quadrant detector works as a two-quadrant photodetector and it can be substituted by a $2 Q$-detector. The principal components of the Slit Sensor are presented in Figure 5. The slit width is $0.5 \mathrm{~mm}$ and the distance $L$ is from the slit to the detector.

In general the Slit Sensor is applied to solar trough collectors and linear Fresnel lenses because its sensitivity is only on one axis. Solar troughs are linear collectors employed in solar plant and they are usually placed parallel to the ground. These systems are provided with a unique motor that performs the sun tracking during the whole day.

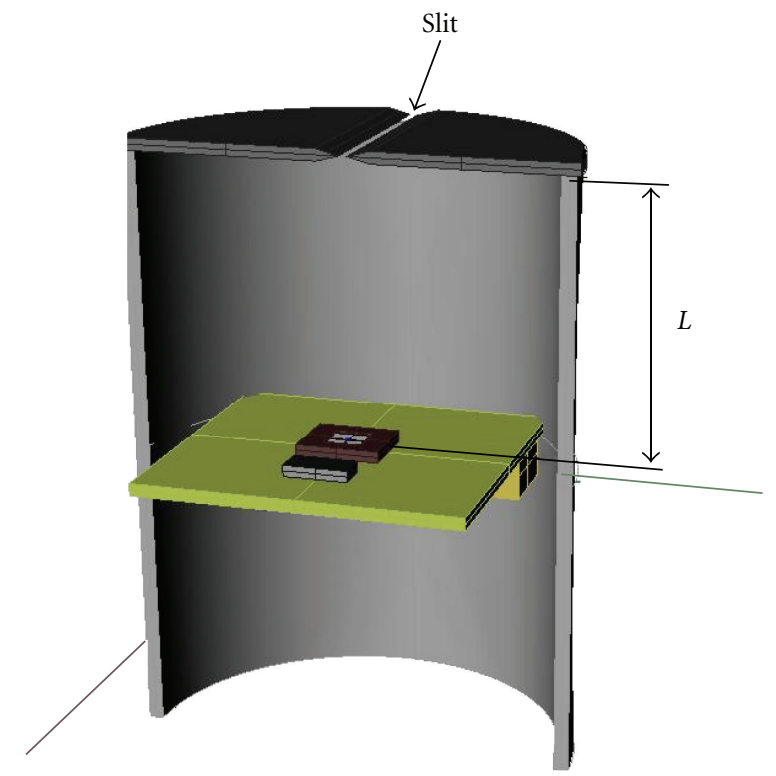

Figure 5: Slit sensor.

The employed sensors of position should be able to work for the entire daytime. Hence they should be sensitive only to the daily movement of the sun and their functioning should not be dependent on the sun's monthly position.

The Slit Sensor developed in our laboratory is based on the projection of a light line produced by a slit. As Figure 6 shows, the light line illuminates the photodetector for every inclination of sun's rays. The figure reports the two extreme cases of sun's ray inclination, corresponding to winter solstice (Dec. 22nd) and to summer solstice (June 21st).

The fine displacements of the sun are assessed, analogously to the case of Pinhole Sensor, from the transversal position of the light line over the photodetector.

\section{Electronics and Signal Elaboration for the Sensors}

The electronic system controlling the motor guide is of digital type. It employs an Input/Output DAQ device NI USB-6009 supplied by National Instrument. The I/O card is 


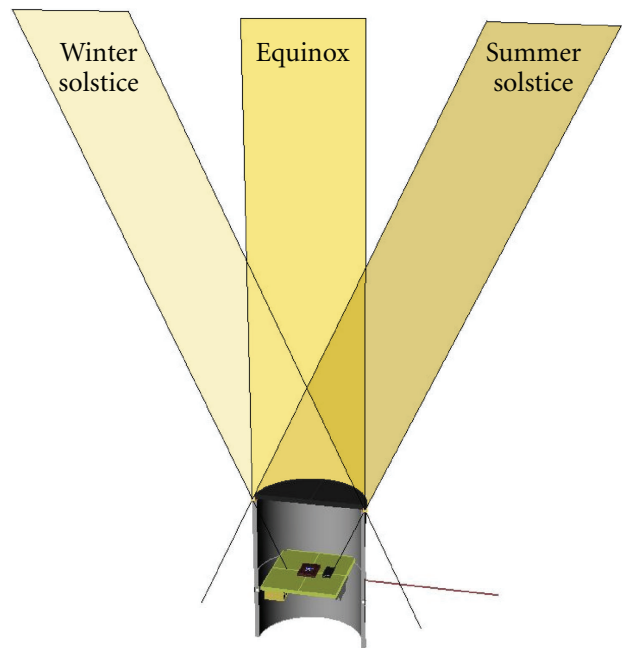

Figure 6: Ray incidence on slit sensor.

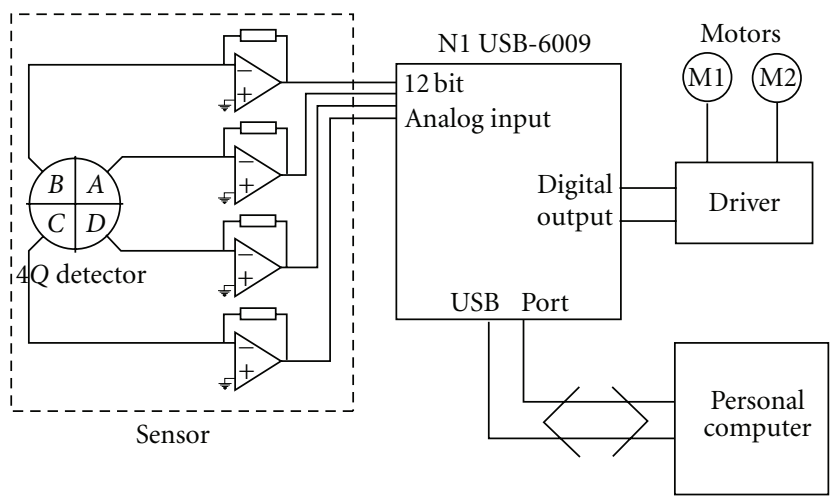

FIGURE 7: Scheme of the electronics.

linked to a personal computer through a connection of USB type. The analogical inputs of the card are used to manage the photodetector with 2 or 4 quadrants of the sun finder. The digital outputs are employed to guide the drivers of electric motors, which move the concentrators or the collectors' supports.

The programme, which assesses the solar ephemerides and emits the correct sequence of pulses to guide the motors, is located in the computer devoted to system management.

Figure 7 presents the electronic scheme, where the sensor components are surrounded by a dashed rectangle. The signals provided by the photodetector are elaborated to obtain the spot misalignment. A suitable algorithm determines the sun's spotdisplacement over the surface of the pointer sensor.

In case of Pinhole Sensor, Figure 8 reports the four quadrants of $4 Q$-detector, indicated as $A, B, C, D$. The circle represents the sun's image over the sensor.

The spot misalignment is expressed by the coordinates of point $P\left(P_{x} ; P_{y}\right)$, which is the barycentre of solar image. $P_{x}$ and $P_{y}$ are the coordinates with respect to the reference axes of detector surface $(X ; Y)$, indicated in Figure 8.

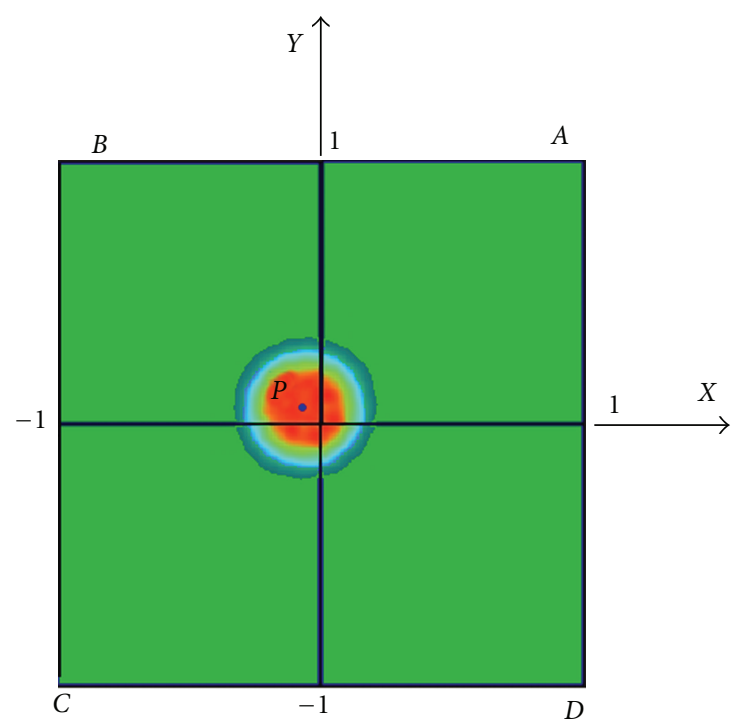

FIgure 8: 4Q-detector with sun's spot.

The misalignment of sun's image barycentre is provided by the following algorithm:

$$
\begin{aligned}
& P_{X}=\frac{\left(V_{A}+V_{D}\right)-\left(V_{B}+V_{C}\right)}{V_{A}+V_{B}+V_{C}+V_{D}}, \\
& P_{Y}=\frac{\left(V_{A}+V_{B}\right)-\left(V_{C}+V_{D}\right)}{V_{A}+V_{B}+V_{C}+V_{D}},
\end{aligned}
$$

where $V_{A}, V_{B}, V_{C}$, and $V_{D}$ are the values (in Volts) of output signals arriving from the four preamplifiers.

The elaboration procedure is analogous for the case of Slit Sensor, but considering only $P_{x}$.

\section{Laboratory Tests of the Sun Finder Sensors}

The laboratory setup to test Pinhole Sensor and Slit Sensor is described in Figure 9. An He-Ne laser beam is expanded to obtain a collimated beam. This light impinges on the sensor under test with different angles of incidence obtained by rotating the sensor. This experimentation has two purposes: the first is to test the angular sensitivity of the sensor and the second is to check the output voltage level. This latter must be sufficiently high with respect to the noise of the electronics; but at the same time the voltage level must guarantee a sufficient dynamic of the sensor.

Figures 10(a)-10(b) illustrate the sensor response measured in laboratory for Pinhole Sensor (Figure 10(a)) and Slit Sensor (Figure 10(b)). The misalignment of image centre is plotted versus the rotation angle representing the angle of incidence of luminous rays. The plotted quantity is the misalignment of sun's image, and it is always defined by (2) or (3). It is the unique misalignment $(P)$ for the Slit Sensor and an exemplificative one $\left(P_{x}\right)$ of the two misalignments for the Pinhole Sensor (the behaviour of the perpendicular one $P_{y}$ is analogous).

Finally Figure 11 combines the results of the laboratory tests on the two sensors, to evidence the difference between 


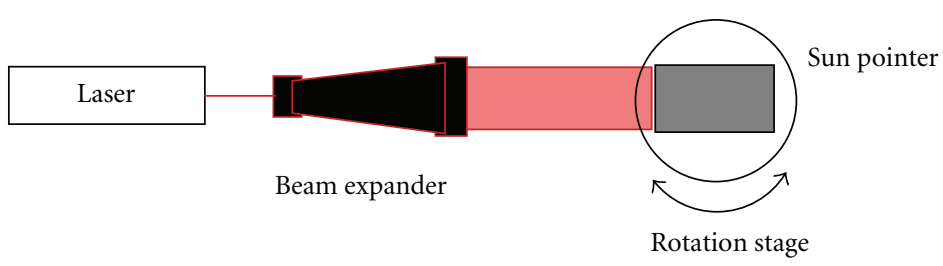

FIgURE 9: Test setup for the laboratory tests of the sensors.

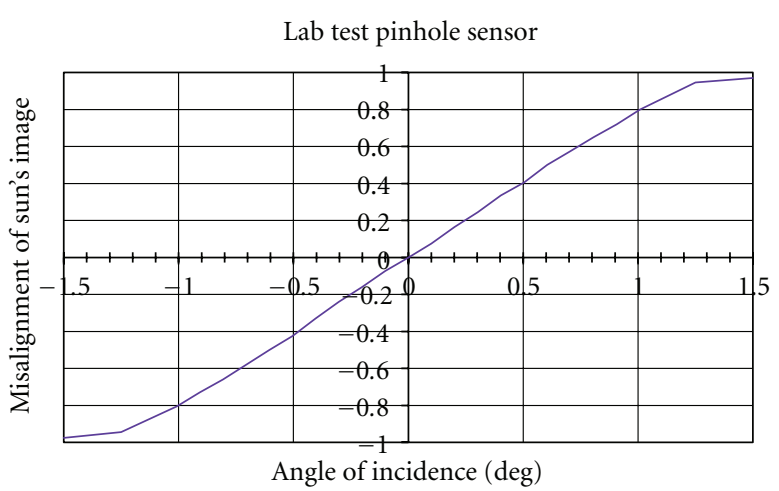

(a)

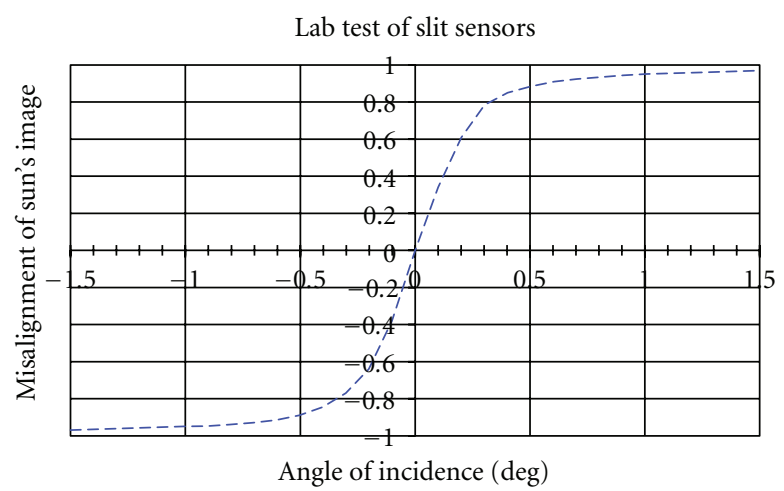

(b)

FIgure 10: (a) Results for Pinhole Sensor. (b) Results for Slit Sensor.

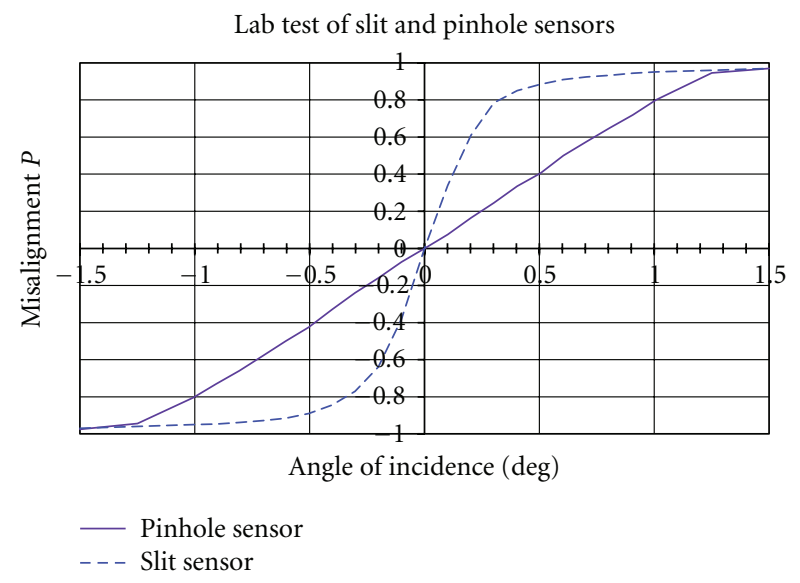

Figure 11: Results of laboratory tests.

curve slopes. Since the two sensors' images have different shape and dimensions, the curves show a different behaviour. The response of Pinhole Sensor saturates for incidence angles of $\pm 1.5^{\circ}$. For the Slit Sensor response, the saturation angles are around $\pm 0.5^{\circ}$.

In conclusion, laboratory tests have assessed that the angular resolution is at least $0.1^{\circ}$, for Pinhole and Slit Sensors. As evidenced by Figure 11, the Pinhole Sensor has higher sensitivity than the Slit Sensor. However, the main advantage of Pinhole Sensor is to work on two axes, while the Slit Sensor is a sun pointing system working only in one direction.

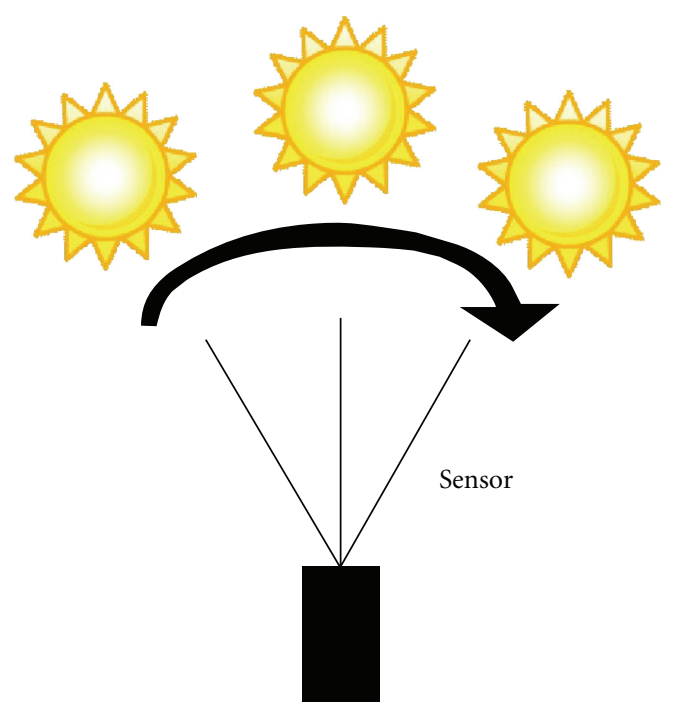

FIGURE 12: Scheme of field tests of the sensors.

\section{Field Tests of the Sun Finder Sensors}

The sun tracking sensors were experimented in operative conditions with direct exposition to the sun. The aim of these field tests was to verify if the sensor characteristics measured in laboratory are applicable also in real conditions.

The tracking system for outdoor tests was placed with the axis aligned to the North-South direction, with a fixed orientation towards the sun, as shown in Figure 12. The signals provided by the sensor were acquired for a time 


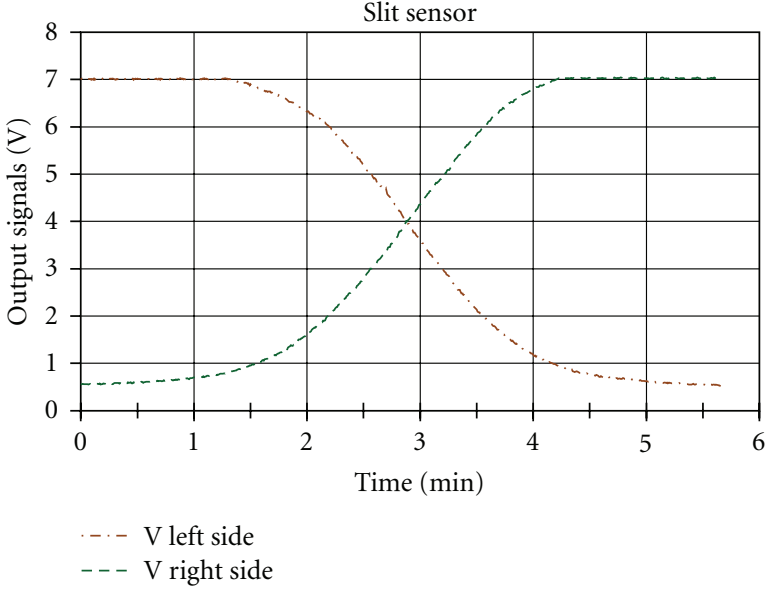

(a)

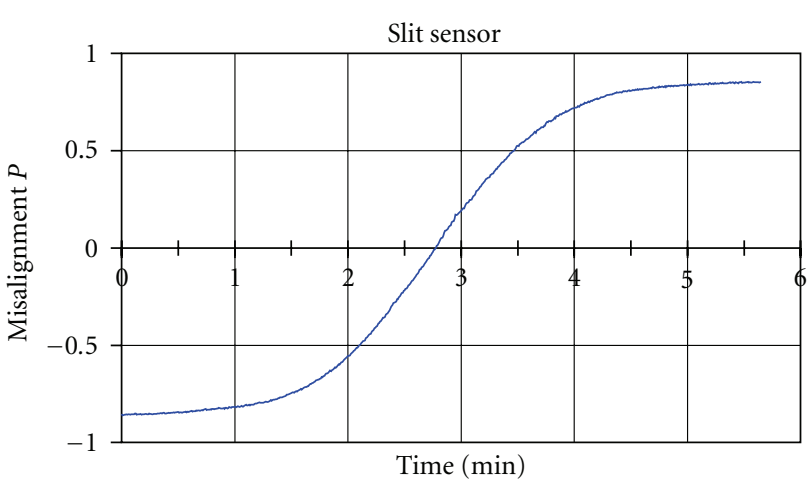

(b)

FIGURE 13: (a) Output signals for Slit Sensor. (b) Misalignment for Slit Sensor.

interval sufficient to detect the sun's passage over the sensor at noontime. For Slit and Pinhole Sensors, the signals were recorded for few minutes every 0.5 second.

Figures 13(a) and 13(b) present the results of field tests performed with the Slit Sensor. Figure 13(a) reports the output signals belonging to the left and right sides of Slit Sensor versus acquisition time. The curves show how the output signals vary in respect to the different sun's positions. Then the sun's misalignment $P$ can be obtained using (2) of Section 5, where $\left(V_{B}+V_{C}\right)$ is the left-side signal and $\left(V_{A}+V_{D}\right)$ is the right-side signal. The resulting curve for the misalignment $P$ is presented in Figure 13(b).

Then the time axis can be converted into angular coordinate, to have more useful graphics and to check the correspondence with the laboratory characterisation of Figures 10 (a) and 10(b). The time-to-angular coordinate transformation is calculated considering that the sun performs a complete rotation of $360^{\circ}$ every 24 hours. Hence the sun covers an angular trajectory of $0.0042^{\circ}$ of arc in a second of time.

For the Slit Sensor, Figure 14 reports laboratory measurement and field test results, expressed as a function of the incidence angle.

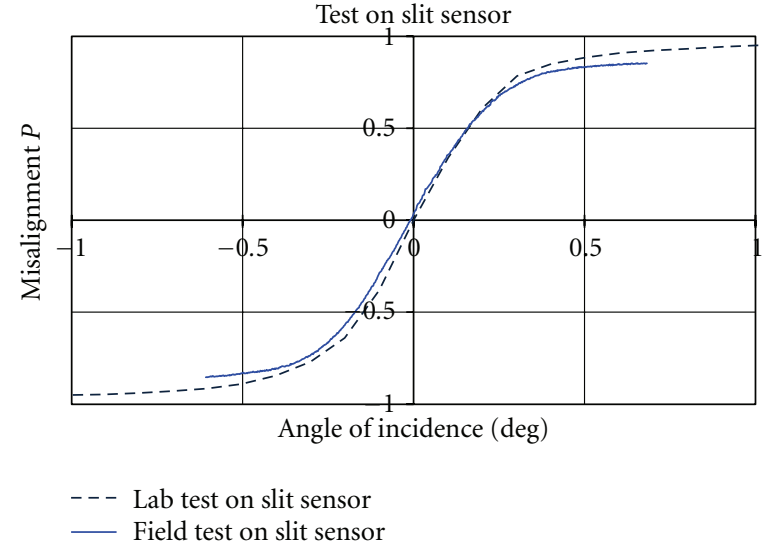

Figure 14: Lab and filed tests for Slit Sensor.

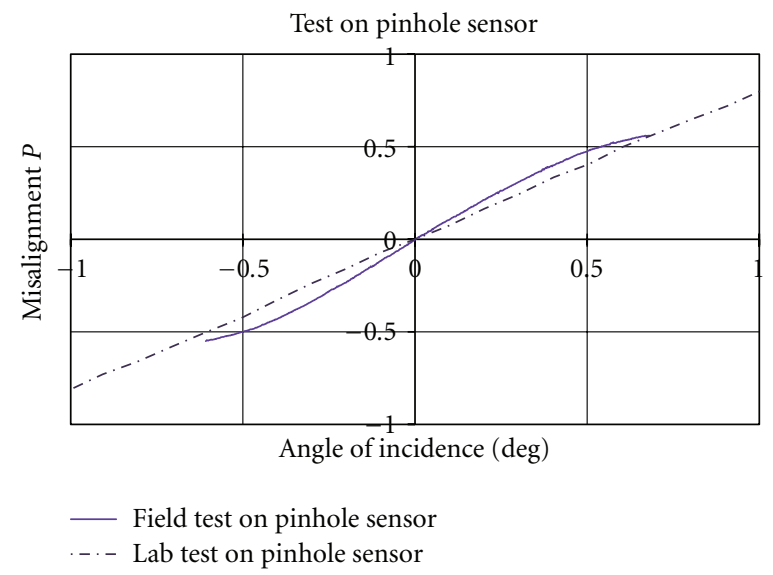

FIgURE 15: Lab and field tests for pinhole sensor.

Laboratory and field measurements for Pinhole Sensor are reported in Figure 15, plotting misalignment versus incidence angle and estimating the match with the curve of Figure 10(a).

The curve measured with direct exposition to the sun is in agreement with the laboratory measurements for all sensors. However, the match is particularly good for the case of Slit Sensor. It is interesting to note that there is perfect coincidence between the two curves in the central zone of Figure 14. The differences at the curve extremes probably depend on the different hardware amplifications used in the two tests. The real sun's detection requires selecting and adjusting the electronic amplification. In the field test, the Slit Sensor has a reduced amplification, so the maximum current value is lower with respect to the laboratory tests.

In conclusion, Figure 16 summarises all the results of laboratory and field tests performed on Pinhole Sensor and Slit Sensor. For all curves in Figure 16, misalignment of sun's rays is plotted versus incidence angle. These curves allow us to verify the amount of variation of sensor response obtained for different angles of incident solar radiation. The lower angular tilt allowed by the laboratory instrumentation was $0.1^{\circ}$, while for the field tests the lower angular tep was $0.002^{\circ}$. 


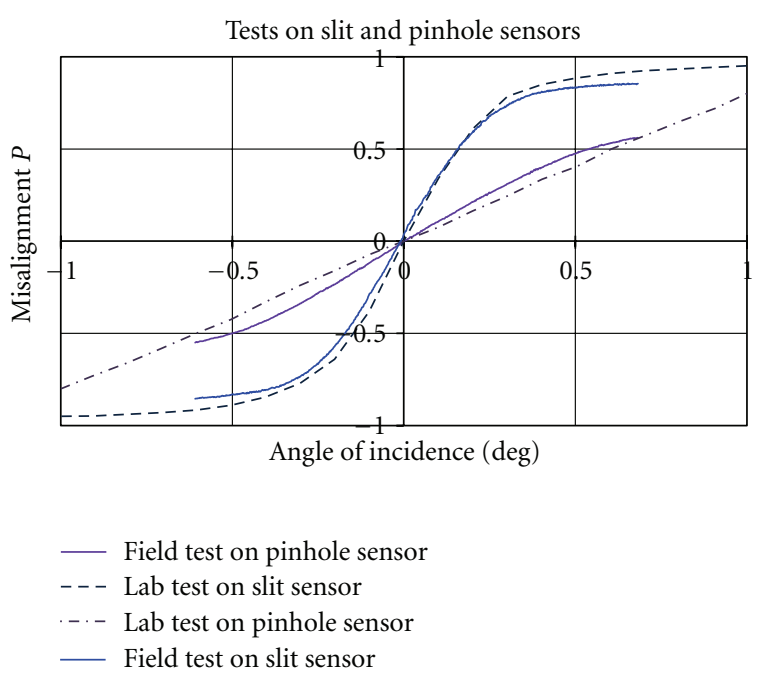

FIGURE 16: Laboratory and field tests for both sensors.

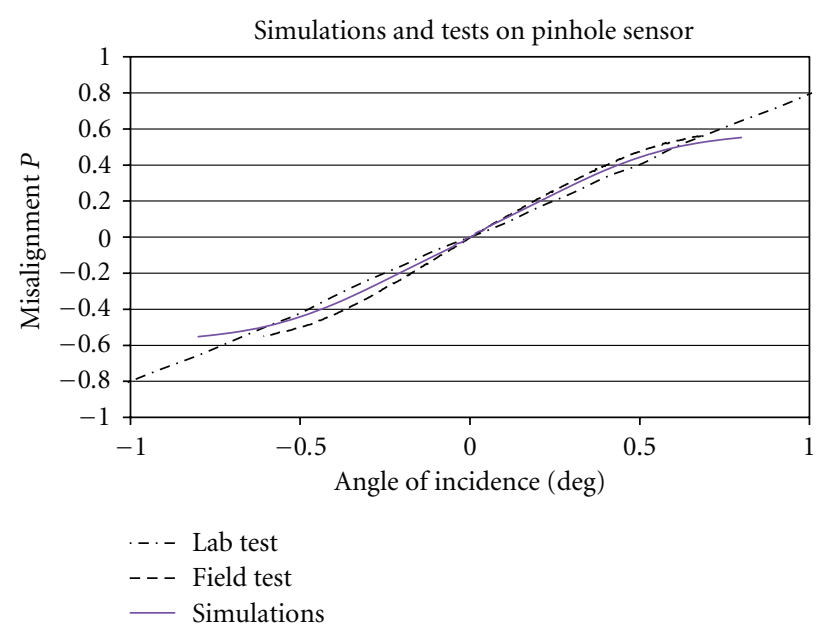

FIGURE 17: Simulated and measured data for pinhole sensor.
The field tests were performed placing the sun pointer towards the sun and acquiring, every 0.5 second, while the sun moved over the pointer. Consequently, applying the time-to-angular coordinate transformation, this temporal sampling corresponded to an angular sampling of $0.002^{\circ}$. From these field tests, the angular resolution results better than $0.01^{\circ}$ for Slit Sensor and Pinhole Sensor. However the Pinhole Sensor remains the most precise one.

In case of long acquisition time, the field test curve can show some disturbs caused by cloud transit. Nevertheless, these isolated perturbations do not affect the sensor functionality.

\section{Theoretical Validation of Tests Results}

The results of the experimentation performed in laboratory and with direct exposition to the sun were finally compared to the corresponding theoretical data. The Pinhole sensor was reproduced in a Zemax-EE optical simulation and the output signal was compared to the signal measured in the laboratory and filed tests. Therefore, the research was completed validating the experimental results by acomparison with the simulated data.

Figure 17 slows the comparison between the output signals obtained for the Pinhole Sensor in the Zemax simulation and in the actual measurements. The output signal is reported as a function of the angle of incidence, as in the previous plots of Figures 14-16. The simulated curve is in fairly good agreement with the data measured in the laboratory and field tests, presented in Figure 15.

\section{Conclusions}

An innovative methodology for sun tracking was developed and experimented both in laboratory and in real working conditions. This tracking procedure is based on an optical pointing system exploiting one or two sensors. The optical tracking system was applied to different testing devices for sunlight collection. This experimentation examined solar concentrators coupled to photovoltaic cells, optical fibres, or linear metal pipes.

The proposed tracking technique consists in a double guiding system: the first one provides the preliminary orientation, then the second one realises the fine positioning and adjustments. The first directing system is of passive type and it drives the motors to correctly orient the collector every day of the year. The second driving system is of dynamic type and it employs an optical sun finder.

The heart of the active tracking system is the sun finder, which is a camera without lenses composed of a Pinhole Sensor (or a Slit Sensor) mounted over a four-quadrant detector. For safety the sun finder usually contains two sections with different Field of Views. The two sections are used in sequence, improving the precision, from the wideangle section to the narrow-angle section.

The distance from the pinhole to the four-quadrant detector determines the sensor FOV aperture. The described optical system can track the sun's position with an angular precision up to $0.01^{\circ}$, but usually the required precision is $0.1^{\circ}$. This angular resolution was experimentally determined for every sensor; moreover, the field tests evidenced that the Pinhole Sensor is more precise than the Slit Sensor.

The tracking system is simple, reliable, and adaptable to all weather conditions and environmental variations. Even in case of sun shading or temporary sun absence, the system provides a realignment of the collector in few seconds. The system is able to compensate possible errors in the placement of the device, which should be correctly aligned to the Earth axis. Figure 18 shows the sun finder with 2 sections, presented in Figures 3(a) and 3(b), mounted and ready to operate. It is usually placed at the centre of the support holding the optical collectors; the third aperture is closed, and a covering 


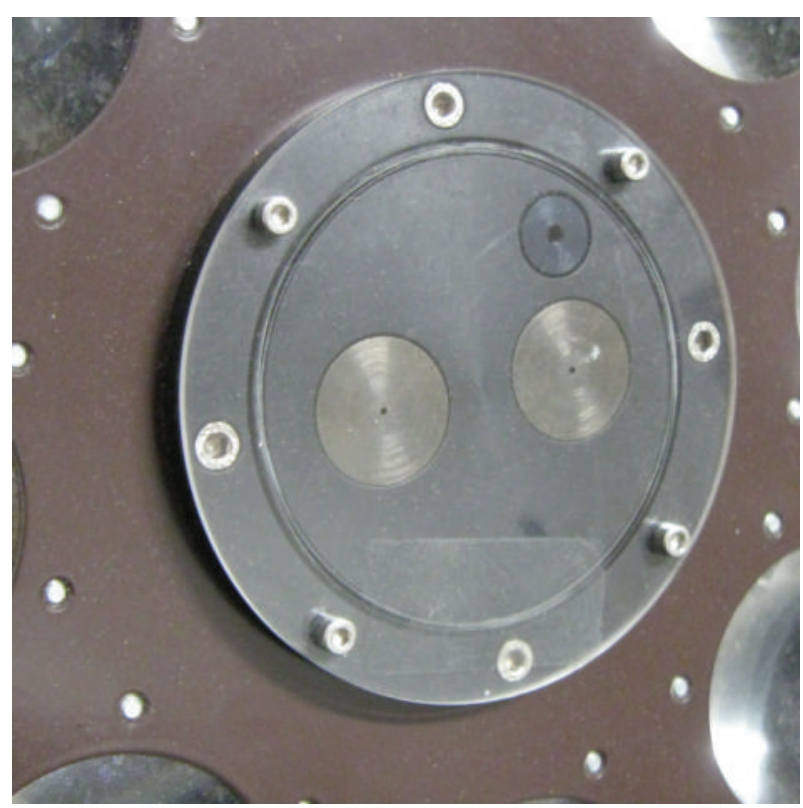

FIGURE 18: The sun finder with 2 sections mounted with the covering glass.

glass protects the pinholes from water and dust. The protection is also essential to preserve the electronics from humidity or other intrusions that could alter its functioning.

Two exemplificative pointing sensors were realised and experimented: Pinhole Sensor and Slit Sensor. The Pinhole Sensor contains a photodetector coupled to a pinhole, while in the Slit Sensor it is coupled to a slit. Suitable mechanics and electronics were realised for the prototypes of both sensors to obtain their optical characterisation. All devices were analysed in laboratory, under controlled and reproducible conditions. Then the sun tracking sensors were experimented in operating conditions, with direct exposition to the sun.

The Pinhole Sensor is the most precise one and it performs sun tracking in two perpendicular directions achieving a very high sensitivity. It is indicated for solar optical systems requiring high pointing precision, like concentrators coupled to small PV cells or optical fibres. The Slit Sensor reaches the same precision of the Pinhole Sensor but only in one direction. Hence it can be applied to linear collectors for solar thermal plants or low concentration CPV systems.

\section{References}

[1] K. K. Chong and C. W. C. Wong, "General formula for onaxis sun-tracking system and its application in improving tracking accuracy of solar collector," Solar Energy, vol. 83, no. 3, pp. 298-305, 2009.

[2] Y. T. Chen, B. H. Lim, and C. S. Lim, "General sun tracking formula for heliostats with arbitrarily oriented axes," Journal of Solar Energy Engineering, vol. 128, no. 2, pp. 245-250, 2006.

[3] S. Armstrong and W. G. Hurley, "Investigating the effectiveness of maximum power point tracking for a solar system," in Proceedings of the 36th IEEE Power Electronics Specialists Conference (PESC'05), pp. 204-209, Recife, Brazil, June 2005.
[4] Y. J. Huang, B. C. Wu, C. Y. Chen, C. H. Chang, and T. C. Kuo, "Solar tracking fuzzy control system design using FPGA," in Proceedings of the World Congress on Engineering (WCE '09), vol. 1, London, UK, July 2009.

[5] Altera Technical Staff, "Build an intelligent solar tracking system with FPGAs," FPGA Sources-EETimes India, 2009, http:// www.powerdesignindia.co.in/STATIC/PDF/200901/PDIOL_ 2009JAN28_GREEN_TA_01.pdf?SOURCES=DOWNLOAD.

[6] C. Y. Lee, P. C. Chou, C. M. Chiang, and C. F. Lin, "Sun tracking systems: a review," Sensors, vol. 9, no. 5, pp. 38753890, 2009.

[7] R. I. Salawu and T. A. Oduyemi, "An electronic sun finder and solar tracking system," Solar \& Wind Technology, vol. 3, no. 3, pp. 215-218, 1986.

[8] Matthias, "A collection of solar finder designs," DD1US, Telescope Reviews: Solar Tips and Tutorials, 2006, http://www .cloudynights.com.

[9] C. Ciamberlini, F. Francini, G. Longobardi, M. Piattelli, and P. Sansoni, "Solar system for exploitation of the whole collected energy," Optics and Lasers in Engineering, vol. 39, no. 2, pp.'233-246, 2003.

[10] F. Francini, D. Fontani, D. Jafrancesco, L. Mercatelli, and P. Sansoni, "Solar internal lighting using optical collectors and fibres," in Nonimaging Optics and Efficient Illumination Systems III, vol. 6338 of Proceedings of SPIE, San Diego, Calif, USA, August 2006, 63380O.

[11] D. Fontani, F. Francini, D. Jafrancesco, G. Longobardi, and P. Sansoni, "Optical design and development of fibre coupled compact solar collectors," Lighting Research \& Technology, vol. 39, no. 1, pp. 17-30, 2007.

[12] D. Fontani, P. Sansoni, F. Francini, L. Mercatelli, and D. Jafrancesco, "A pinhole camera to track the sun position," in Proceedings of the ISES Solar World Congress 2007, Beijing, China, September 2007, t5.1.O12.

[13] D. Fontani, F. Francini, and P. Sansoni, "Optical characterisation of solar concentrator," Optics and Lasers in Engineering, vol. 45, no. 3, pp. 351-359, 2007.

[14] P. Sansoni, F. Francini, D. Fontani, L. Mercatelli, and D. Jafrancesco, "Indoor illumination by solar light collectors," Lighting Research \& Technology, vol. 40, no. 4, pp. 323-332, 2008.

[15] D. Rouan, P. Riaud, A. Boccaletti, Y. Clénet, and A. Labeyrie, "The four-quadrant phase-mask coronagraph. I. Principle," Publications of the Astronomical Society of the Pacific, vol. 112, no. 777, pp. 1479-1486, 2000.

[16] P. Sansoni, D. Fontani, F. Francini, L. Mercatelli, G. Chiani, and M. De Lucia, "Placement and orientation of solar linear troughs," in Proceedings of the 1st International Conference on Solar Heating, Cooling and Buildings (EUROSUN'08), Lisbon, Portugal, October 2008. 


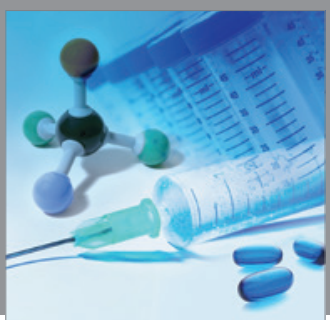

International Journal of

Medicinal Chemistry

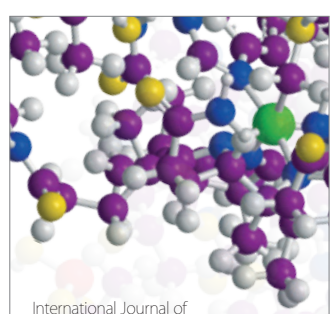

Carbohydrate Chemistry

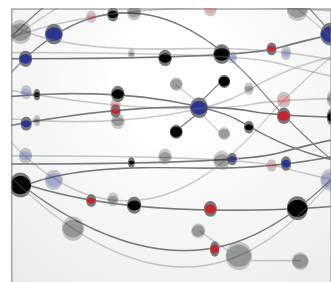

The Scientific World Journal
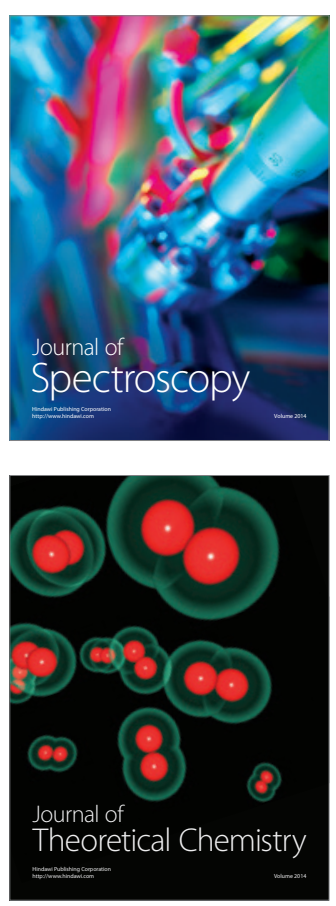
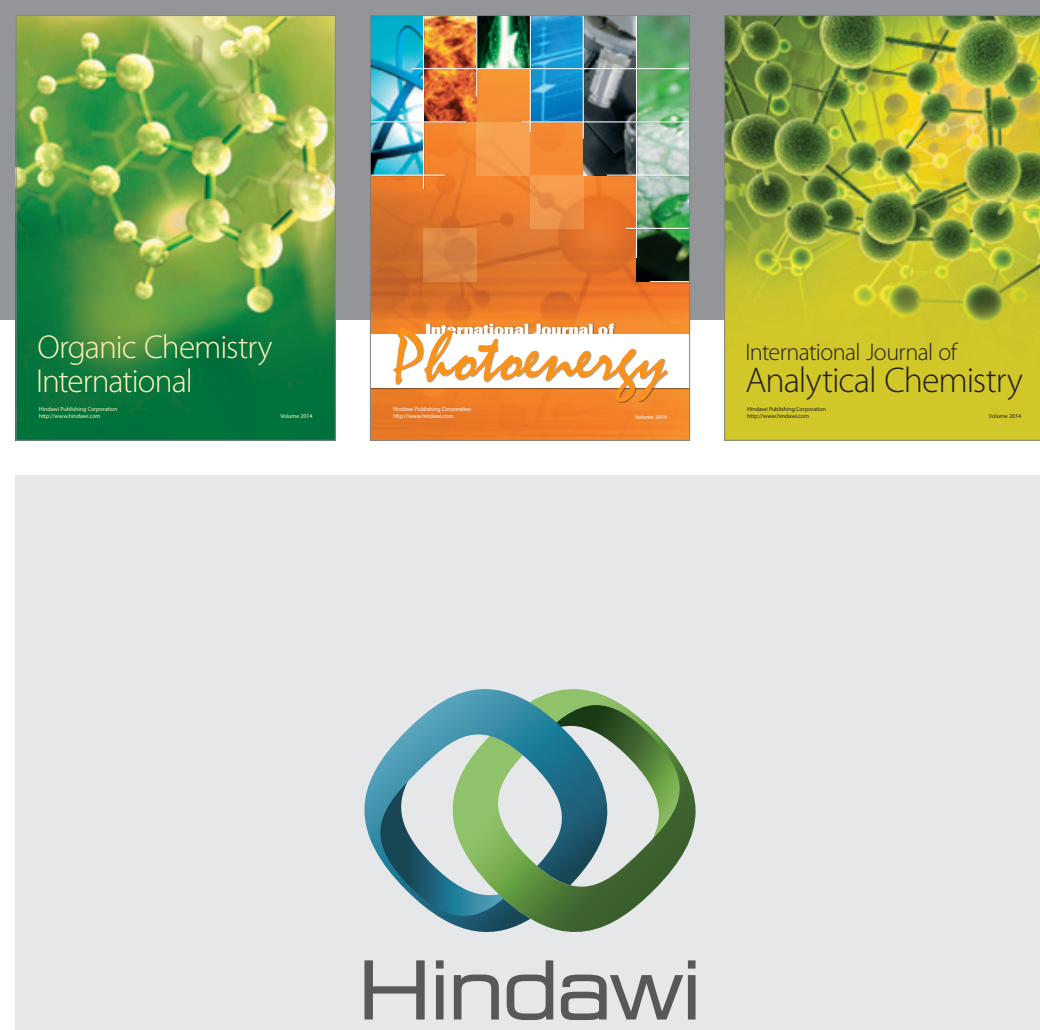

Submit your manuscripts at

http://www.hindawi.com
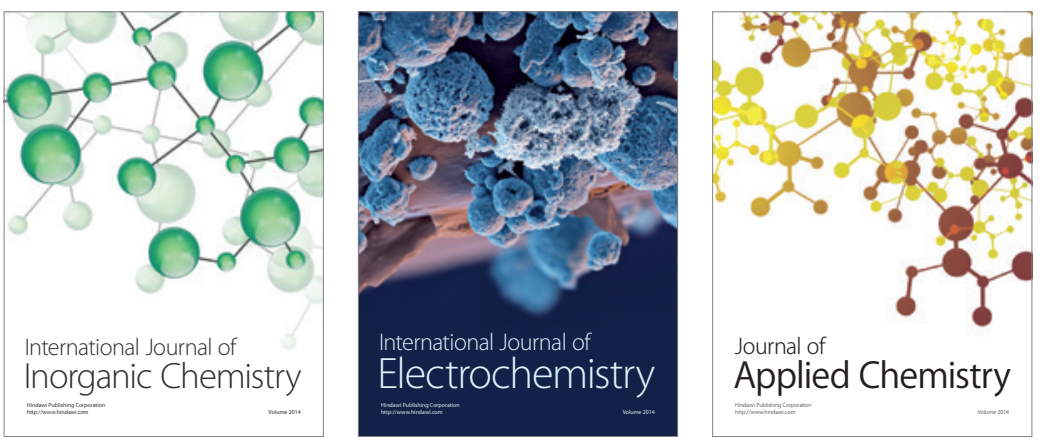

Journal of

Applied Chemistry
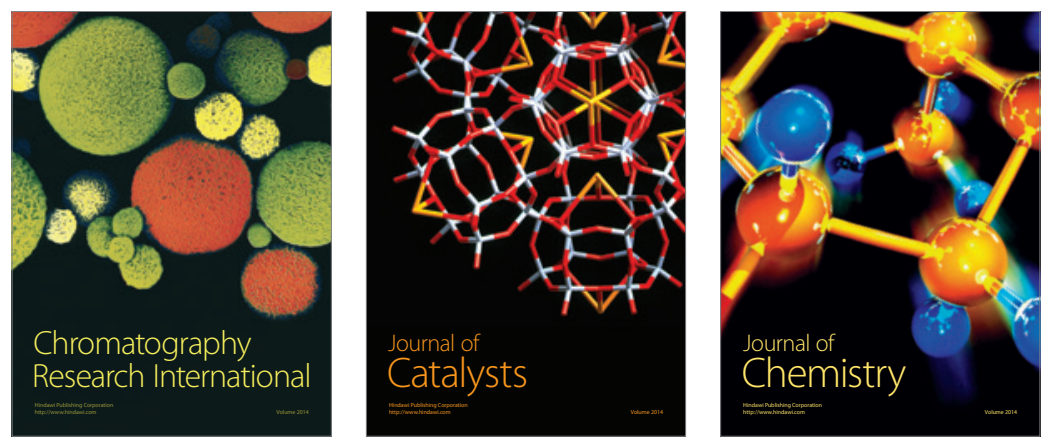
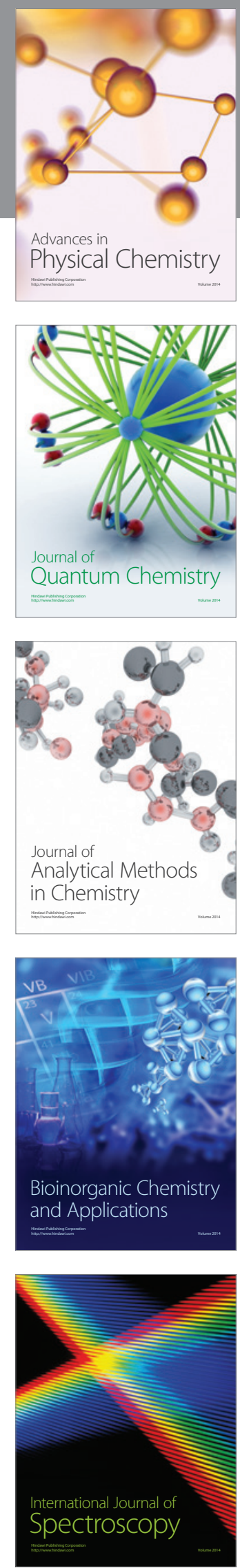